JOURNAL OF INTEGRAL EQUATIONS

AND APPLICATIONS

Volume 17, Number 4, Winter 2005

\title{
MARCINKIEWICZ FUNCTIONS ALONG FLAT SURFACES WITH HARDY SPACE KERNELS
}

\author{
AHMAD AL-SALMAN
}

\begin{abstract}
In this paper, we study Marcinkiewicz integral operators along subvarieties determined by flat surfaces with kernels in the Hardy space $\mathbf{H}^{1}\left(\mathbf{S}^{n-1}\right)$. We establish the $L^{p}$ boundedness of our operators under weak convexity assumptions on the surfaces. Moreover, we establish the $L^{p}$ boundedness of the corresponding Marcinkiewicz integral operators that are related to area integral and Littlewood-Paley $g_{\lambda}^{*}$ functions. Our results offer substantial improvements of previously known results.
\end{abstract}

1. Introduction and statement of results. Let $\mathbf{R}^{n}, n \geq 2$, be the $n$-dimensional Euclidean space and $\mathbf{S}^{n-1}$ the unit sphere in $\mathbf{R}^{n}$ equipped with the induced Lebesgue measure $d \sigma$. Let $\Omega$ be a homogeneous function of degree zero on $\mathbf{R}^{n}$ that is integrable on $\mathbf{S}^{n-1}$ and satisfies

$$
\int_{\mathbf{S}^{n-1}} \Omega(y) d \sigma(y)=0
$$

For a suitable mapping $\Phi: \mathbf{R}^{n} \rightarrow \mathbf{R}^{n}$, consider the Marcinkiewicz integral operator

$$
\mu_{\Omega, \Phi} f(x)=\left(\int_{-\infty}^{\infty}\left|F_{\Omega, \Phi, t}(x)\right|^{2} 2^{-2 t} d t\right)^{1 / 2}
$$

where

$$
F_{\Omega, \Phi, t}(x)=\int_{|y| \leq 2^{t}} f(x-\Phi(y))|y|^{1-n} \Omega(y) d y .
$$

Received by the editors in November, 2003, and in revised form in August, 2005. 2000 AMS Mathematics Subject Classification. Primary 42B20, Secondary 42B15, 42B25.

Key words and phrases. Marcinkiewicz integrals, rough kernels, flat curves, Fourier transform, area integral, Littlewood-Paley $g_{\lambda}^{*}$ functions.

Copyright (C2005 Rocky Mountain Mathematics Consortium 
For the sake of simplicity, we denote $\mu_{\Omega, \Phi}=\mu_{\Omega}$ if $\Phi(y)=y$. The operator $\mu_{\Omega}$ was introduced by E.M. Stein [15]. In [15], Stein proved that if $\Omega$ is continuous and satisfies a $\operatorname{Lip}_{\alpha}(0<\alpha \leq 1)$ condition on $\mathbf{S}^{n-1}$, then $\mu_{\Omega}$ is bounded on $L^{p}$ for all $1<p \leq 2$ and is of weak type $(1,1)$. Subsequently, Benedek, Calderón, and Panzone proved the $L^{p}$ boundedness of $\mu_{\Omega}$ for all $1<p<\infty$ provided that $\Omega$ is continuously differentiable on $\mathbf{S}^{n-1}$ [4]. In [8], Ding, Fan and Pan proved that the operator $\mu_{\Omega}$ is bounded on $L^{p}$ for all $1<p<\infty$ provided that $\Omega \in \mathbf{H}^{1}\left(\mathbf{S}^{n-1}\right)$, the Hardy space on $\mathbf{S}^{n-1}$ (in the sense of Coifman and Weiss [6]; see also [7]). Concerning the operators $\mu_{\Omega, \Phi}$ for general mappings $\Phi$, there has been notable progress in obtaining $L^{p}$ boundedness results provided that the mappings $\Phi$ satisfy $\partial^{\alpha} \Phi / \partial y^{\alpha}(0) \neq 0$ for some multi-index $\alpha \in \mathbf{N}^{n}([\mathbf{3}, \mathbf{5}, \mathbf{8}]$, among others). For mappings $\Phi$ with $\partial^{\alpha} \Phi / \partial y^{\alpha}(0)=0$ for all multi-indices $\alpha \in \mathbf{N}^{n}$, very little is known about the $L^{p}$ boundedness of $\mu_{\Omega, \Phi}[\mathbf{1}]$.

The primary focus of our investigation is the $L^{p}$ boundedness of the operators $\mu_{\Omega, \Phi}$ when $\Phi$ is allowed to be very flat in the sense that $\partial^{\alpha} \Phi / \partial y^{\alpha}(0)=0$ for all multi-indices $\alpha \in \mathbf{N}^{n}$ and $\Omega$ is rough in $\mathbf{H}^{1}\left(\mathbf{S}^{n-1}\right)$. To be more specific, we focus our attention in this paper on the operators $\mu_{\Omega, \Phi}$ when $\Phi(y)=\varphi(|y|) y^{\prime}$, where $\varphi: \mathbf{R}^{+} \rightarrow \mathbf{R}$ is a suitable nonnegative function. In what follows, we shall simply denote $\mu_{\Omega, \Phi}$ by $\mu_{\Omega, \varphi}$. We remark here that one of our main concerns is to obtain the $L^{p}$ boundedness of $\mu_{\Omega, \varphi}$ without imposing heavy conditions like "finite doubling time condition" $\varphi^{\prime}(C t) \geq \varphi^{\prime}(t)$ or "growth condition" $\varphi(2 t) \leq c \varphi(t)$. In fact, we shall study $\mu_{\Omega, \varphi}$ under much weaker conditions. In stating our main results, we shall be considering nonnegative $\mathcal{C}^{1}$ functions $\varphi: \mathbf{R}^{+} \rightarrow \mathbf{R}$ that satisfy the following weak convexity conditions:

$$
\begin{aligned}
& A(\varphi)=\inf _{r>0} \frac{r \varphi^{\prime}(r)}{\varphi(r)}>0 \\
& B(\varphi)=\inf _{r>0} \frac{\varphi(2 r)}{\varphi(r)}>1 .
\end{aligned}
$$

Model examples for functions $\varphi$ that satisfy (1.4) and (1.5) are $t^{\alpha}(\alpha>0)$, real valued polynomials on $\mathbf{R}$ with positive coefficients, $\mathcal{C}^{2}$ strictly increasing convex functions $\varphi$ with $\varphi(0)=0$. Amazingly, a lot of flat functions satisfy the conditions (1.4)-(1.5). For example, 
we cite the function $\varphi(t)=t^{2} e^{-1 / t^{2}}$. Moreover, there are many nonconvex functions that satisfy (1.4)-(1.5). It is worth pointing out that, many authors have considered the conditions (1.4)-(1.5) in their study of some singular integral operators and oscillatory singular integral operators ([10, 12], among others). But in most cases one of the two heavy conditions; "finite doubling time condition" and "growth condition," were added as an extra condition.

Our first result is the following.

Theorem A. Suppose that $\varphi: \mathbf{R}^{+} \rightarrow \mathbf{R}$ is a strictly increasing function with $\varphi(0)=0$ and satisfying the convexity conditions (1.4)-(1.5). If $\Omega \in \mathbf{H}^{1}\left(\mathbf{S}^{n-1}\right)$ and satisfying (1.1), then the operator $\mu_{\Omega, \varphi}$ is bounded on $L^{p}\left(\mathbf{R}^{n}\right)$ for $1<p<\infty$.

As a consequence of Theorem A, we obtain the $L^{p}$ boundedness of the parametric Marcinkiewicz integral operators introduced by Hörmander in 1960 [14] (for more results see [9]). Our result below gives an answer for a long standing open problem, as pointed out in [9].

Theorem B. Suppose that $\rho>0$, and let $\Omega \in \mathbf{H}^{1}\left(\mathbf{S}^{n-1}\right)$ satisfy (1.1). Then the parametric Marcinkiewicz integral operator

$$
\mu_{\Omega}^{\rho} f(x)=\left(\left.\left.\int_{-\infty}^{\infty}\left|2^{-\rho t} \int_{|y| \leq 2^{t}} f(x-y)\right| y\right|^{-n+\rho} \Omega(y) d y\right|^{2} d t\right)^{1 / 2}
$$

is bounded on $L^{p}\left(\mathbf{R}^{n}\right)$ for $1<p<\infty$.

It should be pointed out here that Ding, Lu and Yabuta studied the operator $\mu_{\Omega}^{\rho}$ and showed that it is bounded on $L^{2}\left(\mathbf{R}^{n}\right)$ provided that $\Omega \in \mathbf{L} \log \mathbf{L}\left(\mathbf{S}^{n-1}\right)[\mathbf{9}]$. Therefore, since $\mathbf{L} \log \mathbf{L}\left(\mathbf{S}^{n-1}\right) \varsubsetneqq \mathbf{H}^{1}\left(\mathbf{S}^{n-1}\right)$, Theorem B gives a substantial improvement of Ding, Lu and Yabuta's result as far as the operator $\mu_{\Omega}^{\rho}$ is concerned.

Concerning the result in Theorem A, it can be generalized as follows. 
Theorem C. Let $\varphi: \mathbf{R}^{+} \rightarrow \mathbf{R}$ be a strictly increasing function with $\varphi(0)=0$ and satisfying the convexity conditions (1.4)-(1.5). Let $\Omega \in \mathbf{H}^{1}\left(\mathbf{S}^{n-1}\right)$ and satisfying (1.1). For each $\lambda \in \mathbf{R}$, define the operator $\mu_{\Omega, \varphi, \lambda}$ by

$$
\begin{aligned}
& \mu_{\Omega, \varphi, \lambda} f(x) \\
= & \left(\left.\left.\int_{-\infty}^{\infty}\left|\int_{|y| \leq 2^{t}} f\left(x-\lambda \varphi(|y|) y^{\prime}\right)\right| y\right|^{-n+1} \Omega\left(y^{\prime}\right) d y\right|^{2} 2^{-2 t} d t\right)^{1 / 2} .
\end{aligned}
$$

Then the operators $\left\{\mu_{\Omega, \varphi, \lambda}\right\}_{\lambda \in \mathbf{R}}$ are uniformly bounded on $L^{p}\left(\mathbf{R}^{n}\right)$ for $1<p<\infty$.

Also, in this paper, we shall establish the following $L^{p}$ boundedness result of the corresponding Marcinkiewicz integral operators that are related to area integral and Littlewood-Paley $g_{\lambda}^{*}$ functions.

Theorem D. Suppose that $\varphi: \mathbf{R}^{+} \rightarrow \mathbf{R}$ is strictly increasing function with $\varphi(0)=0$ and satisfying the convexity conditions (1.4)-(1.5). If $\Omega \in \mathbf{H}^{1}\left(\mathbf{S}^{n-1}\right)$ and satisfying (1.1), then for $2 \leq p<\infty$ and $s>1$, the operators $\widetilde{\mu}_{\Omega, \varphi}$ and $\mu_{\Omega, \varphi, s}^{*}$ satisfy

$$
\begin{aligned}
\left\|\tilde{\mu}_{\Omega, \varphi}(f)\right\|_{p} & \leq C_{p}\|f\|_{p} \\
\left\|\mu_{\Omega, \varphi, s}^{*}(f)\right\|_{p} & \leq C_{p}\|f\|_{p},
\end{aligned}
$$

where

$$
\begin{aligned}
\tilde{\mu}_{\Omega, \varphi} f(x) & =\left(\int_{\Upsilon(x)}\left|F_{\Omega, \varphi, t}(z)\right|^{2} 2^{-(2+n) t} d z d t\right)^{1 / 2}, \\
\Upsilon(x) & =\left\{(z, t) \in \mathbf{R}^{n+1}:|x-z|<2^{t}\right\}
\end{aligned}
$$

$$
=\left(\iint_{\mathbf{R}^{n+1}}\left(2^{t}\left(2^{t}+|x-z|\right)^{-1}\right)^{n s}\left|F_{\Omega, \varphi, t}(z)\right|^{2} 2^{-(2+n) t} d z d t\right)^{1 / 2},
$$

and $F_{\Omega, \varphi, t}$ is given by (1.3) with $\Phi(y)=\varphi(|y|) y^{\prime}$. 
Remarks. (1) It is well known that, for $1<q \leq \infty$ and $0<\alpha<1$ the relations

$$
\begin{aligned}
\mathcal{C}^{1}\left(\mathbf{S}^{n-1}\right) & \varsubsetneqq \operatorname{Lip}_{\alpha}\left(\mathbf{S}^{n-1}\right) \varsubsetneqq \mathbf{L} \log \mathbf{L}\left(\mathbf{S}^{n-1}\right) \varsubsetneqq \mathbf{H}^{1}\left(\mathbf{S}^{n-1}\right) \\
& \varsubsetneqq L^{1}\left(\mathbf{S}^{n-1}\right)
\end{aligned}
$$

hold. Therefore, our condition on the function $\Omega$ in Theorems A-D is the weakest among all above function spaces on $\mathbf{S}^{n-1}$.

(2) It should be noticed that if $\varphi$ is allowed to be very flat at the origin (which is of course our case), then the singularity of $\mu_{\Omega, \varphi}$ at the origin will be too severe. Therefore, to obtain the $L^{p}$ boundedness of $\mu_{\Omega, \varphi}$, we have to work a little bit harder than in the case for the classical Marcinkiewicz integral operator. In fact, due to the flatness of the surface and the absence of the finite doubling time condition or even the growth condition, specially constructed square functions are needed.

This paper is organized as follows. In Section 2, we shall review the definition of the Hardy space $\mathbf{H}^{1}\left(\mathbf{S}^{n-1}\right)$. In Section 3, we establish some preliminary estimates. In Section 4 , we shall prove Theorem A, Theorem B, Theorem C and Theorem D.

Finally, throughout this paper, the letter $C$ is a positive constant that may vary at each occurrence but it is independent of the essential variables. Also, we shall use $\exp (\cdot)$ to denote $e^{(\cdot)}$.

The Hardy space $\mathbf{H}^{1}\left(\mathbf{S}^{n-1}\right)$. In this section, we recall the definition of the hardy space $\mathbf{H}^{1}\left(\mathbf{S}^{n-1}\right)$. The Hardy space $\mathbf{H}^{1}\left(\mathbf{S}^{n-1}\right)$ can be defined by using atoms:

Definition 2.1. A function $\mathbf{a}: \mathbf{S}^{n-1} \rightarrow \mathbf{C}$ is called an $\mathbf{H}^{1}$ atom if it satisfies the following:

(i) $\operatorname{supp}(\mathbf{a}) \subseteq \mathbf{S}^{n-1} \cap\left\{y \in \mathbf{R}^{n}:\left|y-y_{0}\right|<\rho\right\}$ for some $y_{0} \in \mathbf{S}^{n-1}$ and $\rho>0$;

(ii) $\|\mathbf{a}\| \leq \rho^{-(n-1)}$;

(iii) $\int_{\mathbf{S}^{n-1}} \mathbf{a}(x) d \sigma(x)=0$. 
For the sake of simplicity, we shall refer to $\rho$ and $y_{0}$ in the above definition by $\operatorname{rad}(\mathbf{a})$ and cent $(\mathbf{a})$, respectively.

Definition 2.2. A function $\Omega: \mathbf{S}^{n-1} \rightarrow \boldsymbol{C}$ is in $\mathbf{H}^{1}\left(\mathbf{S}^{n-1}\right)$ if there are $\mathbf{H}^{1}$ atoms $\mathbf{a}_{1}, \mathbf{a}_{2}, \ldots$, on $\mathbf{S}^{n-1}$, a sequence of complex numbers $\left\{c_{j}\right\}$ with $\sum_{j}\left|c_{j}\right|<\infty$, and $\Omega_{0} \in L^{\infty}$ such that

$$
\boldsymbol{\Omega}=\Omega_{0}+\sum_{j} c_{j} \mathbf{a}_{j}
$$

Here, $\sum_{j}\left|c_{j}\right| \leq\|\Omega\|_{\mathbf{H}}^{1}\left(\mathbf{S}^{n-1}\right)$.

3. Preparation. Given a strictly increasing function $\varphi: \mathbf{R}^{+} \rightarrow \mathbf{R}$ with $\varphi(0)=0$ and satisfying the convexity conditions (1.4)-(1.5). For a homogeneous function $\Omega$ of degree zero on $\mathbf{R}^{n}$ that is integrable on $\mathbf{S}^{n-1}$ and satisfies (1.1), define the function $H_{\Omega, \varphi}$ on $\mathbf{R} \times R^{n}$ by

$$
H_{\Omega, \varphi}(\xi, t)=\frac{1}{2^{t}} \int_{2^{t-1} \leq|y|<2^{t}} \exp \left(-i\left(\xi \cdot \varphi(|y|) y^{\prime}\right)\right) \Omega\left(y^{\prime}\right)|y|^{1-n} d y
$$

Then, we have the following:

Lemma 3.1. If $\Omega$ is an $\mathbf{H}^{1}$ atom on $\mathbf{S}^{n-1}$ with $\operatorname{cent}(\Omega)=y_{0}=$ $(0, \ldots, 1)$ and $\operatorname{rad}(\Omega)=\rho$. Then

(i) $\left|H_{\Omega, \varphi}(\xi, t)\right| \leq C\left|\varphi\left(2^{t-1}\right)\right| \rho^{2} \xi||^{-\beta}$ for $0<\rho<1 / n$ and $\xi \in \mathbf{R}^{n}$.

(ii) $\left|H_{\Omega, \varphi}(\xi, t)\right| \leq C\left|\varphi\left(2^{t-1}\right)\right| \rho \xi^{0} \|^{-\beta}$ for $0<\rho<1 / n$ and $\xi^{0} \in$ $\mathbf{R}^{n-1}$ with $\xi=\left(\xi^{0}, 0\right)$.

(iii) $\left|H_{\Omega, \varphi}(\xi, t)\right| \leq C\left|\varphi\left(2^{t-1}\right)\right| \xi||^{-\beta}$ for $\rho \geq 1 / n$ and $\xi \in \mathbf{R}^{n}$.

Here $C$ is a constant independent of $\rho, \xi$ and $t$.

Proof. Let $g$ be the real valued function that is defined on $\mathbf{S}^{n-1} \times$ $\mathbf{S}^{n-1} \times \mathbf{S}^{n-1}$ by $g\left(\xi^{\prime}, y^{\prime}, z^{\prime}\right)=\xi^{\prime} \cdot\left(y^{\prime}-z^{\prime}\right)$. Then it is straightforward to show that

$$
\sup _{\xi^{\prime} \in \mathbf{S}^{n-1}} \int_{\operatorname{Supp}(\Omega)}\left|g\left(\xi^{\prime}, y^{\prime}, z^{\prime}\right)\right|^{-1 / 4 n} d \sigma\left(y^{\prime}\right) \leq C \rho^{(n-1)}\left|\rho^{2}\right|^{-1 / 4 n}
$$




$$
\sup _{\substack{\xi^{\prime} \in \mathbf{S}^{n-1}, \xi^{\prime} \cdot y_{0}=0}} \int_{\operatorname{Supp}(\Omega)}\left|g\left(\xi^{\prime}, y^{\prime}, z^{\prime}\right)\right|^{-1 / 4 n} d \sigma\left(y^{\prime}\right) \leq C \rho^{(n-1)}|\rho|^{-1 / 4 n}
$$

for $0<\rho<1 / n$, where $C$ is a constant independent of $\rho[\mathbf{2}]$. Here, $\operatorname{Supp}(\Omega)$ denotes the support of the function $\Omega$.

Now, it is easy to see that

$$
\left|H_{\Omega, \varphi}(\xi, t)\right|^{2} \leq C \rho^{-2(n-1)} \int_{\operatorname{Supp}(\Omega)} \int_{\operatorname{Supp}(\Omega)} I\left(t, \xi^{\prime}, y^{\prime}, z^{\prime}\right) d \sigma\left(y^{\prime}\right) d \sigma\left(z^{\prime}\right),
$$

where

$$
I\left(t, \xi^{\prime}, y^{\prime}, z^{\prime}\right)=\left|\int_{1}^{2} \exp \left(-i\left(|\xi| \varphi\left(2^{t-1} r\right)\left(\xi^{\prime} \cdot\left(y^{\prime}-\cdot z^{\prime}\right)\right)\right)\right) d r\right| .
$$

By (1.4) and an application of the van der Corput lemma [17], we obtain

$$
I\left(t, \xi^{\prime}, y^{\prime}, z^{\prime}\right) \leq \min \left\{1,\{A(\varphi)\}^{-1}|| \xi\left|\varphi\left(2^{t}\right) g\left(\xi^{\prime}, y^{\prime}, z^{\prime}\right)\right|^{-1}\right\} .
$$

Thus, by (3.5), (3.4), (3.2), (3.3) and the observation $|\operatorname{Supp}(\Omega)|=$ $C \rho^{n-1}$, we immediately obtain (i) and (ii).

The proof of (iii) is straightforward. In fact, by (3.5) and condition (ii) in Definition 2.1, we get

$$
\begin{aligned}
\left|H_{\Omega, \varphi}(\xi, t)\right| & \leq \rho^{-(n-1)} \int_{\mathbf{S}^{n-1}} I\left(t, \xi, y^{\prime}, 0\right) d \sigma\left(y^{\prime}\right) \\
& \leq \rho^{-(n-1)}\{A(\varphi)\}^{-1}|| \xi\left|\varphi\left(2^{t}\right)\right|^{-1 / 2 n} \int_{\mathbf{S}^{n-1}}\left|\xi^{\prime} \cdot y^{\prime}\right|^{-1 / 2 n} \\
& \leq C|| \xi\left|\varphi\left(2^{t}\right)\right|^{-1 / 2 n},
\end{aligned}
$$

where the last inequality follows by the fact that $\rho \geq 1 / n$ and the boundedness of the integral in the second inequality in (3.6). This completes the proof of the lemma.

Now, for $\xi \in \mathbf{R}^{n}$, consider the maximal function

$$
M_{\varphi, \xi} f(x)=\sup _{t \in \mathbf{R}}\left|\frac{1}{2^{t}} \int_{0}^{2^{t}} f(x-\varphi(r) \xi) d r\right| .
$$


Clearly, if $\xi=0$, then $M_{\varphi, \xi}$ reduces to the identity operator on $\mathbf{R}^{n}$. Therefore, in the rest of this section, we shall assume that $\xi \neq 0$. Our concern here is $L^{p}$ boundedness of the operator $M_{\varphi, \xi}$. If $\varphi$ satisfies an additional condition like $\varphi(t) \leq c \varphi(2 t)$ for all $t>0$ and some fixed $c>0$, then we can use a theorem in [17, p. 477] and establish the desired $L^{p}$ boundedness result, see also [12]. But, obviously, such a condition might not be available in our case. However, this obstacle can be resolved by first noticing that

$$
M_{\varphi, \xi} f(x) \leq 4 \widetilde{M}_{\varphi, \xi} f(x)=\sup _{j \in \mathbf{Z}}\left|\int_{2^{j+1}}^{2^{j+1}}\right| f(x-\varphi(r) \xi)\left|\frac{d r}{r}\right| .
$$

Then, by a well-known bootstrapping argument $[\mathbf{1 0}, \mathbf{1 1}]$, we obtain the $L^{p}$ boundedness of $\widetilde{M}_{\varphi, \xi}$ for all $1<p<\infty$ with $L^{p}$ bounds independent of $\xi$, for more details see [2]. Hence, by this and (3.8), we obtain

$$
\left\|M_{\varphi, \xi}(f)\right\|_{p} \leq C_{p}\|f\|_{p}
$$

for all $1<p<\infty$ with constant $C_{p}$ independent of $\xi$.

Finally, we end this section by the following relation between the operators $\mu_{\Omega, \varphi}$ and $\mu_{\Omega, \varphi, s}^{*}$, which can be obtained by following a similar argument as in the proof of Lemma 4.1 in [8].

Lemma 3.2. Let $s>1$. Then, for any nonnegative function $g$, we have

$$
\int_{\mathbf{R}^{n}}\left(\mu_{\Omega, \varphi, s}^{*} f(x)\right)^{2} g(x) d x \leq C_{s} \int_{\mathbf{R}^{n}}\left(\mu_{\Omega, \varphi} f(x)\right)^{2}(H g)(x) d x
$$

where $H$ is the classical Hardy-Littlewood maximal operator on $\mathbf{R}^{n}$.

Proof. The proof is simple. In fact, the inequality (3.10) is an immediate consequence of the definition of $\mu_{\Omega, \varphi, s}^{*}$ in (1.6) and the following simple inequality:

$$
\sup _{t \in \mathbf{R}} 2^{-n t} \int_{\mathbf{R}^{n}}\left(\frac{2^{t}}{2^{t}+|x-y|}\right)^{n s} g(x) d x \leq C_{s}(H g)(y) .
$$


4. Proof of main results. In this section, we prove Theorem B and Theorem C. In order to do so, we start by proving the following:

Lemma 4.1. Let $\varphi: \mathbf{R}^{+} \rightarrow \mathbf{R}$ be a strictly increasing function with $\varphi(0)=0$ and satisfying the convexity conditions (1.4)-(1.5). Let $\Omega$ be an $\mathbf{H}^{1}$ atom on $\mathbf{S}^{n-1}$ with $\operatorname{Cent}(\Omega)=y_{0}=(0, \ldots, 1)$ and $\operatorname{rad}(\Omega)=\rho<1 / n$. For $t \in \mathbf{R}$, let $\Lambda(t)=\left\{y \in \mathbf{R}^{n}: 2^{t} \leq|y|<2^{t}\right\}$. For a fixed $\kappa \in \mathbf{Z}$, let $\mu_{\Omega, \varphi, \lambda}^{\kappa}$ be the operator given by

$$
\begin{aligned}
& \mu_{\Omega, \varphi, \lambda}^{\kappa}(f)(x) \\
& =\left(\int_{-\infty}^{\infty}\left|\frac{1}{2^{t+\kappa}} \int_{\Lambda(t+\kappa)} f\left(x-\lambda \varphi(|y|) y^{\prime}\right) \frac{\Omega\left(y^{\prime}\right)}{|y|^{n-1}} d y\right|^{2} d t\right)^{1 / 2} .
\end{aligned}
$$

Then

$$
\left\|\mu_{\Omega, \varphi, \lambda}^{\kappa}(f)\right\|_{p} \leq C_{p}\|f\|_{p}
$$

for all $1<p<\infty$, where $C_{p}$ independent of $\rho, \lambda$ and $\kappa$.

Proof. For $t \in \mathbf{R}$, let $\sigma_{t, \varphi, \lambda}$ and $\nu_{t, \varphi, \lambda}$ be the measures defined on the Fourier transform side by

$$
\begin{aligned}
\hat{\sigma}_{t, \varphi, \lambda}(\xi) & =H_{\Omega, \lambda \varphi}(\xi, t) \\
\hat{\nu}_{t, \varphi, \lambda}(\xi) & =H_{\Omega, \lambda \varphi}(\bar{\xi}, t),
\end{aligned}
$$

where, $\bar{\xi}=\left(\xi_{1}, \ldots, \xi_{n-1}, 1\right)$ for $\xi=\left(\xi_{1}, \ldots, \xi_{n-1}, \xi_{n}\right)$ and $H_{\Omega, \lambda \varphi}$ is given by (3.1) with $\varphi$ replaced by $\lambda \varphi$. Then, it is clear that

$$
\mu_{\Omega, \varphi, \lambda}^{\kappa}(f)(x)=\left(\int_{-\infty}^{\infty}\left|\sigma_{t+\kappa, \varphi, \lambda} * f(x)\right|^{2} d t\right)^{1 / 2} .
$$

Moreover, by the definitions of $\sigma_{t, \varphi, \lambda}$ and $\nu_{t, \varphi, \lambda}$, it follows

$$
\left\|\sigma_{t+\kappa, \varphi, \lambda}\right\| \leq 1 \quad \text { and } \quad\left\|\nu_{t+\kappa, \varphi, \lambda}\right\| \leq 1 .
$$

Now, by the cancelation property (iii) in Definition 2.1, we immediately obtain

$$
\hat{\sigma}_{t+\kappa, \varphi, \lambda}(0)=\widehat{\nu}_{t+\kappa, \varphi, \lambda}(0)=0 .
$$


By Lemma 3.1, there exist constants $0<\beta<1$ and $C>0$ independent of $\rho, \kappa, t$ and $\xi$ such that

$$
\begin{aligned}
\left|\hat{\sigma}_{t+\kappa, \varphi, \lambda}(\xi)\right| & \leq\left. C\left|\varphi\left(2^{t+\kappa-1}\right)\right| \lambda \rho^{2} \xi\right|^{-\beta} \\
\left|\hat{\nu}_{t+\kappa, \varphi, \lambda}(\xi)\right| & \leq C\left|\varphi\left(2^{t+\kappa-1}\right)\right| \lambda \rho \bar{\xi}||^{-\beta} .
\end{aligned}
$$

On the other hand, by the cancelation property (iii), we have

$$
\begin{aligned}
\left|\hat{\sigma}_{t+\kappa, \varphi, \lambda}(\xi)-\hat{\nu}_{t+\kappa, \varphi, \lambda}(\xi)\right| & \leq C\left|\varphi\left(2^{t+\kappa}\right)\right| \lambda \rho^{2} \xi|| \\
\left|\hat{\nu}_{t+\kappa, \varphi, \lambda}(\xi)\right| & \leq C\left|\varphi\left(2^{t+\kappa}\right)\right| \lambda \rho \bar{\xi}|| .
\end{aligned}
$$

Let $\sigma_{\varphi, \lambda, \kappa}^{*}$ and $\nu_{\varphi, \lambda, \kappa}^{*}$ be the operators given by

$$
\sigma_{\varphi, \lambda, \kappa}^{*}(f)(x)=\sup _{t \in \mathbf{R}}\left|\sigma_{t+\kappa, \varphi, \lambda} * f(x)\right|
$$

and

$$
\nu_{\varphi, \lambda, \kappa}^{*}(f)(x)=\sup _{t \in \mathbf{R}}\left|\nu_{t+\kappa, \varphi, \lambda} * f(x)\right| .
$$

Then by Hölder's inequality, the fact that $\|\Omega\|_{L^{1}} \leq 1$, and (3.9), we obtain

$$
\begin{gathered}
\left\|\sigma_{\varphi, \lambda, \kappa}^{*}(f)\right\|_{p} \leq C_{p}\|f\|_{p} \\
\left\|\nu_{\varphi, \lambda, \kappa}^{*}(f)\right\|_{p} \leq C_{p}\|f\|_{p}
\end{gathered}
$$

for all $1<p<\infty$ with constant $C_{p}$ independent of $\kappa$ and $\lambda$.

Now choose $\theta \in C_{0}^{\infty}(\mathbf{R})$ such that $\theta(t)=1$ for $|t| \leq 1 / 2$ and $\theta(t)=0$ for $|t| \geq 1$. Let $\left\{\vartheta_{t+\kappa, \varphi, \lambda}: t \in \mathbf{R}\right\}$ and $\left\{\digamma_{t+\kappa, \varphi, \lambda}: t \in \mathbf{R}\right\}$ be the families of measures given by

$$
\begin{aligned}
& \widehat{\vartheta}_{\mathrm{t}+\kappa, \varphi, \lambda}(\xi)=\widehat{\nu}_{t+\kappa, \varphi, \lambda}(\xi) \theta\left(\left.\left|\varphi\left(2^{t+\kappa-1}\right)\right| \lambda \rho^{2} \xi\right|^{2}\right) \\
& \widehat{\digamma}_{\mathrm{t}+\kappa, \varphi, \lambda}(\xi)=\widehat{\sigma}_{t+\kappa, \varphi, \lambda}(\xi)-\widehat{\vartheta}_{t+\kappa, \varphi, \lambda}(\xi) .
\end{aligned}
$$


Then, by (4.6), (4.7)-(4.10), (4.13)-(4.14), and the properties of $\theta$, we have

$$
\begin{aligned}
\left|\widehat{\digamma}_{t+\kappa, \varphi, \lambda}(\xi)\right| & \leq\left. C\left|\varphi\left(2^{t+\kappa-1}\right)\right| \lambda \rho^{2} \xi\right|^{-\alpha} ; \\
\left|\widehat{\digamma}_{t+\kappa, \varphi, \lambda}(\xi)\right| & \leq\left. C\left|\varphi\left(2^{t+\kappa}\right)\right| \lambda \rho^{2} \xi\right|^{\alpha} ; \\
\left|\widehat{\vartheta}_{t+\kappa, \varphi, \lambda}(\xi)\right| & \leq C\left|\varphi\left(2^{t+\kappa-1}\right)\right| \lambda \rho \bar{\xi} \|^{-\alpha} ; \\
\left|\widehat{\vartheta}_{t+\kappa, \varphi, \lambda}(\xi)\right| & \leq C\left|\varphi\left(2^{t+\kappa-1}\right)\right| \lambda \rho \bar{\xi} \|^{\alpha} ; \\
\left\|\digamma_{t+\kappa, \varphi, \lambda}\right\|\left\|\vartheta_{t+\kappa, \varphi, \lambda}\right\| & \leq C,
\end{aligned}
$$

where $C$ is a constant independent of $\rho, \xi, t, \lambda$ and $\kappa$. Moreover, by (4.11)-(4.12) and (4.13)-(4.14), we have

$$
\begin{gathered}
\left\|\digamma_{\varphi, \lambda, \kappa}^{*}(f)\right\|_{p} \leq C_{p}\|f\|_{p} \\
\left\|\vartheta_{\varphi, \lambda, \kappa}^{*}(f)\right\|_{p} \leq C_{p}\|f\|_{p}
\end{gathered}
$$

for all $1<p<\infty$ with constant $C_{p}$ independent of $\kappa$ and $\lambda$.

Now since

$$
\sigma_{t+\kappa, \varphi, \lambda}=\digamma_{t+\kappa, \varphi, \lambda}+\vartheta_{t+\kappa, \varphi, \lambda},
$$

we immediately obtain

$$
\mu_{\Omega, \varphi, \lambda}^{\kappa}(f)(x) \leq \mu_{\Omega, \varphi, \lambda}^{\kappa, 1}(f)+\mu_{\Omega, \varphi, \lambda}^{\kappa, 2}(f),
$$

where $\mu_{\Omega, \varphi, \lambda}^{\kappa, 1}$ and $\mu_{\Omega, \varphi, \lambda}^{\kappa, 2}$ are the operators that are given by (4.5) with $\sigma_{t+\kappa, \varphi, \lambda}$ is replaced by $\digamma_{t+\kappa, \varphi, \lambda}$ and $\vartheta_{t+\kappa, \varphi, \lambda}$, respectively.

Since

$$
\left\|\mu_{\Omega, \varphi, \lambda}^{\kappa}(f)\right\|_{p} \leq\left\|\mu_{\Omega, \varphi, \lambda}^{\kappa, 1}(f)\right\|_{p}+\left\|\mu_{\Omega, \varphi, \lambda}^{\kappa, 2}(f)\right\|_{p},
$$

it suffices to prove (4.2) for the operators $\mu_{\Omega, \varphi, \lambda}^{\kappa, 1}$ and $\mu_{\Omega, \varphi, \lambda}^{\kappa, 2}$. We shall prove (4.2) for $\mu_{\Omega, \varphi, \lambda}^{\kappa, 1}$ while the proof for $\mu_{\Omega, \varphi, \lambda}^{\kappa, 2, \lambda}$ is similar. To do so, we argue as follows. 
By an elementary procedure, choose a collection of $\mathcal{C}^{\infty}$ functions $\left\{\omega_{k}\right\}_{k \in \mathbf{Z}}$ on $(0, \infty)$ with the following properties:

(a) $\operatorname{supp}\left(\omega_{k}\right) \subseteq\left[1 / \varphi\left(2^{k+1}\right), 1 / \varphi\left(2^{k-1}\right)\right]$;

(b) $0 \leq \omega_{k} \leq 1$;

(c) $\left|d^{s} \omega_{k} / d u^{s}(u)\right| \leq C_{s} / u^{s}$;

(d) $\sum_{k \in \mathbf{Z}} \omega_{k}(u)=1$.

Define the functions $\left\{\psi_{k}: k \in \mathbf{Z}\right\}$ on $\mathbf{R}^{n}$ by $\hat{\psi}_{k}(y)=\omega_{k}\left(\rho^{2}|\lambda y|\right)$. Then, by property (d), we have

$$
\mu_{\Omega, \varphi, \lambda}^{\kappa, 1}(f)(x) \leq \sum_{k \in \mathbf{Z}} I_{\kappa, k}(f)(x),
$$

where

$$
I_{\kappa, k}(f)(x)=\left(\int_{-\infty}^{\infty}\left|f * \digamma_{t+\kappa, \varphi, \lambda} * \psi_{\lfloor t+\kappa\rfloor+k}(x)\right|^{2} d t\right)^{1 / 2} .
$$

Here, $\lfloor x\rfloor$ is the greatest integer function less than or equal to $x$. As in [1], for $k \in \mathbf{Z}$, let $S_{k}^{\kappa}$ be the operator given by

$$
S_{k}^{\kappa} f(x)=\left(\int_{-\infty}^{\infty}\left|f * \psi_{\lfloor t+\kappa\rfloor+k}(x)\right|^{2} d t\right)^{1 / 2} .
$$

Then, by the properties of $\left\{\psi_{j}\right\}$ and a well-known argument, see [17, p. 46] and [16, pp. 245-246], it is easy to show that

$$
\left\|S_{k}^{\kappa}(f)\right\|_{p} \leq C\|f\|_{p}
$$

for all $p \in(1, \infty)$ with constant $C$ depends only on $p$ and the dimension of the underlying space $\mathbf{R}^{n}$.

Next, for $p>2$, set $q=(p / 2)^{\prime}$ and choose a nonnegative function $v \in L_{+}^{q}\left(\mathbf{R}^{n}\right)$ with $\|v\|_{q}=1$ such that

$$
\left\|I_{\kappa, k}(f)\right\|_{p}^{2}=\int_{R^{n}} \int_{-\infty}^{\infty}\left|f * \digamma_{t+\kappa, \varphi, \lambda} * \psi_{\lfloor t+\kappa\rfloor+k}(x)\right|^{2} v(x) d t d x .
$$

Therefore, we have

$$
\begin{aligned}
\left\|I_{\kappa, k}(f)\right\|_{p} & \leq\left\|S_{k}^{\kappa}(f)\right\|_{p}\left\|\digamma_{\varphi, \lambda, \kappa}^{*}(v)\right\|_{q}^{1 / 2} \\
& \leq C\|f\|_{p},
\end{aligned}
$$


where the last inequality follows by (4.20) and (4.26).

By duality, we get

$$
\left\|I_{\kappa, k}(f)\right\|_{(p)^{\prime}} \leq C\|f\|_{(p)^{\prime}} .
$$

In conclusion, we get

$$
\left\|I_{\kappa, k}(f)\right\|_{p} \leq C\|f\|_{p}
$$

for all $1<p<\infty$.

Now, we estimate $\left\|I_{\kappa, k}(f)\right\|_{2}$. For $l \in \mathbf{Z}$ and nonzero $\xi \in \mathbf{R}^{n}$, let $E_{l}(\xi)$ be the interval in $\mathbf{R}$ given by

$$
E_{l}(\xi)=\left[\log _{2}\left(2^{l-1} \varphi^{-1}\left(|\xi|^{-1}\right)\right), \log _{2}\left(2^{l+2} \varphi^{-1}\left(\|\left.\xi\right|^{-1}\right)\right)\right] .
$$

It is radially seen that

$$
\left|E_{l}(\xi)\right|=3 .
$$

Moreover, for $k, \kappa \in \mathbf{Z}$ and nonzero $\xi \in \mathbf{R}^{n}$, we have

$$
\varphi\left(2^{-k-1} \varphi^{-1}\left(|\xi|^{-1}\right)\right) \leq \varphi\left(2^{t+\kappa}\right) \leq \varphi\left(2^{-k+2} \varphi^{-1}\left(|\xi|^{-1}\right)\right)
$$

for all $t \in E_{k-\kappa}(\xi)$. Therefore, for $\kappa \in \mathbf{Z}, \xi \in \mathbf{R}^{n} \backslash\{0\}$, conditions (1.4)-(1.5) imply

$$
\begin{array}{r}
\varphi\left(2^{t+\kappa}\right) \leq\{B(\varphi)\}^{-k+2}|\xi|^{-1} \\
\text { for } k \geq 3 \text { and } t \in E_{k-\kappa}(\xi)
\end{array}
$$

and

$$
\begin{aligned}
& \varphi\left(2^{t+\kappa-1}\right) \geq\{B(\varphi)\}^{-k-2}|\xi|^{-1} \\
& \text { for } \quad k \leq-2 \text { and } t \in E_{k-\kappa}(\xi) .
\end{aligned}
$$

Thus, for $k \geq 3$, by Plancherel's theorem, (4.15), (4.31) and (4.34), we have

$$
\begin{aligned}
\left\|I_{\kappa, k}(f)\right\|_{2}^{2} & \leq C \int_{\mathbf{R}^{n}}|\hat{f}(\xi)|^{2} \int_{-\infty}^{\infty}\left|\varphi\left(2^{t+\kappa}\right)\right| \rho^{2} \lambda \xi||^{2 \alpha} \\
& \times\left|\omega_{\lfloor t+\kappa\rfloor+k}\left(\left|\rho^{2} \lambda \xi\right|\right)\right|^{2} d t d \xi \\
& \leq C\{B(\varphi)\}^{2(-k+3) \alpha} \int_{\mathbf{R}^{n}}|\hat{f}(\xi)|^{2} d \xi
\end{aligned}
$$


Hence,

$$
\left\|I_{\kappa, k}(f)\right\|_{2} \leq C\{B(\varphi)\}^{(-k+2) \alpha}\|f\|_{2} \quad \text { for } \quad k \geq 3 .
$$

Similarly, by Plancherel's theorem, (4.16), (4.31) and (4.34), we have

$$
\left\|I_{\kappa, k}(f)\right\|_{2} \leq C\{B(\varphi)\}^{(k+2) \alpha}\|f\|_{2} \quad \text { for } \quad k \leq-3 .
$$

One more application of Plancherel's theorem along with (4.20) and (4.32), we obtain

$$
\left\|I_{\kappa, k}(f)\right\|_{2} \leq \sqrt{3}\|f\|_{2} \quad \text { for } \quad k=-2,-1,0,1,2 .
$$

Now, by $(4.30),(4.36),(4.37),(4.38)$ and an interpolation argument, we obtain

$$
\left\|I_{\kappa, k}(f)\right\|_{p} \leq C\{B(\varphi)\}^{-|k| \alpha \theta(p)}\|f\|_{p},
$$

for $1<p<\infty$ with constant $C$ independent of $\rho$ and $\kappa$. Hence,

$$
\begin{aligned}
\left\|\mu_{\Omega, \varphi, \lambda}^{\kappa, 1}(f)\right\|_{p} & \leq \sum_{k \in \mathbf{Z}}\left\|I_{\kappa, k}(f)\right\|_{p} \leq C\left\{\sum_{k \in \mathbf{Z}}\{B(\varphi)\}^{-|k| \alpha \theta(p)}\right\}\|f\|_{p} \\
& \leq C_{p}\|f\|_{p}
\end{aligned}
$$

where, $C_{p}, C$ and $\theta(p)$ are constants independent of $\rho$ and $\kappa$. This completes the proof.

By following exactly the same argument as in the proof of Lemma 4.2, we have

Lemma 4.2. Let $\varphi, y_{0}, \rho, \kappa$ and $\mu_{\Omega, \varphi, \lambda}^{\kappa}$ be as in Lemma 4.1. If $\Omega$ is an $L^{\infty}$ function on $\mathbf{S}^{n-1}$ or an $\mathbf{H}^{1}\left(\mathbf{S}^{n-1}\right)$ atom with $\operatorname{rad}(\Omega)=\rho \geq$ $1 / n$, then

$$
\left\|\mu_{\Omega, \varphi, \lambda}^{\kappa}(f)\right\|_{p} \leq C_{p}\|f\|_{p}
$$

for all $1<p<\infty$, where $C_{p}$ is a constant independent of $\lambda$. Moreover, it is also independent of $\rho$ if $\Omega$ is an $\mathbf{H}^{1}\left(\mathbf{S}^{n-1}\right)$ atom. 
Now we are ready to prove Theorem $\mathrm{C}$ which is more general than Theorem A.

Proof of Theorem C. Let $\varphi, \Omega, \lambda$ and $\mu_{\Omega, \varphi, \lambda}$ be as in the statement of Theorem C. Since $\Omega \in \mathbf{H}^{1}\left(\mathbf{S}^{n-1}\right)$, there are $\mathbf{H}^{1}$ atoms $\mathbf{a}_{1}, \mathbf{a}_{2}, \ldots$, on $\mathbf{S}^{n-1}$, a sequence of complex numbers $\left\{c_{j}\right\}$ with $\sum_{j}\left|c_{j}\right|<\infty$, and $\Omega_{0} \in L^{\infty}$ such that

$$
\mathbf{\Omega}=\Omega_{0}+\sum_{j} c_{j} \mathbf{a}_{j}
$$

with $\sum_{j}\left|c_{j}\right| \leq\|\Omega\|_{\mathbf{H}^{1}\left(\mathbf{S}^{n-1}\right)}$. Therefore,

$$
\mu_{\Omega, \varphi, \lambda}(f)(x) \leq \mu_{\Omega_{0}, \varphi, \lambda}(f)(x)+\sum_{j}\left|c_{j}\right| \mu_{\mathbf{a}_{j}, \varphi, \lambda}(f)(x) .
$$

First, by Lemma 4.2, we have

$$
\left\|\mu_{\Omega_{0}, \varphi, \lambda}(f)=\mu_{\Omega, \varphi, \lambda}^{0}(f)\right\|_{p} \leq C_{p}\|f\|_{p}
$$

$L^{p}$ for all $1<p<\infty$. Therefore, to prove the desired $L^{p}$ boundedness of $\mu_{\Omega, \varphi, \lambda}$, it suffices to show that $\mu_{\mathbf{a}_{j}, \varphi, \lambda}$ is bounded on $L^{p}$ for all $1<p<\infty$ and any $\mathbf{H}^{1}$ atom $\mathbf{a}_{j}$ with $\left\|\mu_{\mathbf{a}_{j}, \varphi, \lambda}\right\|_{p}$ independent of $\lambda$ and any particular atom.

Thus, given an $\mathbf{H}^{1}\left(\mathbf{S}^{n-1}\right)$ atom $\mathbf{a}_{j}$. By using a proper rotation on $\mathbf{S}^{n-1}$, we may assume that $\operatorname{cent}\left(\mathbf{a}_{j}\right)=(0, \ldots, 1)$. Now, it is easy to see that

$$
\mu_{\mathbf{a}_{j}, \varphi, \lambda}(f)(x) \leq \sum_{\kappa=0}^{\infty} 2^{-\kappa} \mu_{\mathbf{a}_{j}, \varphi, \lambda}^{-\kappa}(f)(x) .
$$

By Lemmas 4.1 and 4.2 , we have

$$
\left\|\mu_{\mathbf{a}_{j}, \varphi, \lambda}^{-\kappa}(f)\right\|_{p} \leq C\|f\|_{p}
$$

for all $1<p<\infty$ with constant $C$ independent of the atom $\mathbf{a}_{j}$ and $\kappa$. Hence, by this and (4.43), we get

$$
\left\|\mu_{\mathbf{a}_{j}, \varphi, \lambda}(f)\right\|_{p} \leq C\|f\|_{p}
$$


for all $1<p<\infty$ with constant $C$ independent of the atom $\mathbf{a}_{j}$. This completes the proof of Theorem C.

Proof of Theorem B. The result of Theorem B is a simple consequence of Theorem A and a suitable change of variable. We omit the details.

We end this section by presenting a proof of Theorem D.

Proof of Theorem D. We shall follow the same lines of the proof of Theorem 5 in $[\mathbf{8}]$. Since $\widetilde{\mu}_{\Omega, \varphi}(f)(x) \leq C_{s}\left[\mu_{\Omega, \varphi, s}^{*}(f)(x)\right]$, it suffices to prove (1.9). By Lemma 3.2 and Theorem A, we immediately get (1.9) for $p=2$. In order to handle the case $p>2$, we only need to use Lemma 3.2, Theorem A, and the observation that

$$
\left\|\mu_{\Omega, \varphi, s}^{*}(f)\right\|_{p}^{2}=\sup _{\|g\|_{(p / 2)^{\prime}}=1}\left|\int_{\mathbf{R}^{n}}\left(\mu_{\Omega, \varphi, s}^{*}(f)(x)\right)^{2} g(x) d x\right| .
$$

This completes the proof.

\section{REFERENCES}

1. A. Al-Salman, On Marcinkiewicz integrals along flat surfaces, Turkish. J. Math. 29 (2005), 111-120.

2. A. Al-Salman and H. Al-Qassem, Singular integrals along flat curves with kernels in the Hardy Space $H^{1}\left(S^{n-1}\right)$, Proc. of Conf. in Honor of Professor Georgii Litvinchuk, "Factorization, Singular Operators and Related Problems" (Madeira, Portugal), 2002, pp. 1-12.

3. - Integral operators of Marcinkiewicz type, J. Integral Equations Appl. 14 (2002), 343-354.

4. A. Benedek, A. Calderón and R. Panzone, Convolution operators on Banach space valued functions, Proc. Natl. Acad. Sci. 48 (1962), 356-365.

5. J. Chen, D. Fan and Y. Pan, A note on a Marcinkiewicz integral operator, Math. Nachr. 227 (2001), 33-42.

6. R.R. Coifman and G. Weiss, Extensions of Hardy spaces and their use in analysis, Bull. Amer. Math. Soc. 83 (1977), 569-645.

7. L. Colzani, Hardy spaces on sphere, Ph.D. Thesis, Washington University, St. Louis, MO, 1982.

8. Y. Ding, D. Fan and Y. Pan, $L^{p}$ Boundedness of Marcinkiewicz integrals with Hardy space function kernel, Acta. Math. Sinica 16 (2000), 593-600 (English ser.). 
9. Y. Ding, S. Lu and K. Yabuta, A problem on rough parametric Marcinkiewicz functions, J. Austral. Math. Soc. 72 (2002), 13-21.

10. J. Duoandikoetxea and J.L. Rubio de Francia, Maximal and singular integral operators via Fourier transform estimates, Invent. Math. 84 (1986), 541-561.

11. D. Fan and Y. Pan, Singular integrals with rough kernels supported by subvarieties, Amer. J. Math. 119 (1997), 799-839.

12. D. Fan, Y. Pan and D. Yang, A weighted norm inequality for rough singular integrals, Tôhoku Math. J. 51 (1999), 141-161.

13. L. Grafakos and A. Stefanov, $L^{p}$ bounds for singular integrals and maximal singular integrals with rough kernels, Indiana Univ. Math. J. 47 (1998), 455-469. Mr 99i: 42019. Zb1 913.42014.

14. L. Hörmander, Translation invariant operators, Acta Math. 104 (1960), 93-139.

15. E.M. Stein, On the function of Littlewood-Paley, Lusin and Marcinkiewicz, Trans. Amer. Math. Soc. 88 (1958), 430-466.

16. - Singular integrals and differentiability properties of functions, Princeton Univ. Press, Princeton, 1970.

17. - Harmonic analysis: Real-variable methods, orthogonality and oscillatory integrals, Princeton Univ. Press, Princeton, 1993.

Department of Mathematics, Yarmouk University, Irbid-Jordan

E-mail address: alsalman@yu.edu.jo 